\title{
Philonsorbonne
}

$10 \mid 2016$

Année 2015-2016

\section{La personne et ses modes chez Pierre Abélard}

\section{Ángela Beatriz ÁVALOS SOTO}

\section{OpenEdition}

\section{Journals}

Édition électronique

URL : https://journals.openedition.org/philonsorbonne/767

DOI : 10.4000/philonsorbonne.767

ISSN : 2270-7336

\section{Éditeur}

Publications de la Sorbonne

\section{Édition imprimée}

Date de publication : 1 janvier 2016

Pagination : $9-28$

ISSN : 1255-183X

\section{Référence électronique}

Ángela Beatriz ÁVALOS SOTO, «La personne et ses modes chez Pierre Abélard », Philonsorbonne [En ligne], 10 | 2016, mis en ligne le 19 janvier 2016, consulté le 09 juin 2021. URL : http:// journals.openedition.org/philonsorbonne/767 ; DOI : https://doi.org/10.4000/philonsorbonne.767

(c) Tous droits réservés 


\title{
La personne et ses modes chez Pierre Abélard
}

\author{
Ángela Beatriz Ávalos Soto
}

Tribus itaque seu quatuor modis ac pluribus fortassis hoc nomen 'persona' sumitur, aliter videlicet a theologis, aliter a grammaticis, aliter a rhetoricis uel in comoediis, ut supra determinatum est ${ }^{1}$.

La «personne» est une notion plurielle, valable pour le théologien comme pour le grammairien, le rhéteur ou le comédien. De cette citation extraite de la Theologia Christiana d'Abélard surgissent les interrogations suivantes : comment comprendre une telle extension de la persona? Quel sens le mot revêt-il dans ces diverses occurrences? Y a-t-il pour le justifier un dénominateur commun aux différents «agents » concernés, ou n'est-ce qu'une pure homonymie? Enfin, la figure du comédien s'identifie-t-elle à celle du rhéteur?

Pour qui veut répondre avec cohérence, il importe de repérer l'ordre dans lequel les différentes acceptions sont ici présentées. À suivre la phrase, la «personne » est une notion qui concerne d'abord le théologien, puis le grammairien, enfin le rhéteur et le comédien. Ce n'est pas dû au hasard ; cette hiérarchie correspond assez fidèlement à l'architecture globale de la pensée d'Abélard tandis qu'il écrit la Theologia Christiana, contemporaine de son De Intellectibus. La théologie, en effet, comprend les arts du langage, et dans les arts du langage, la grammaire domine la rhétorique, puis la comédie. De façon primordiale, par conséquent, c'est-à-dire dans ses

1. P. Abélard, Theologia Christiana (= TC), III, in Petri Abaelardi Opera Theologica, vol. II, éd. E. Buytaert, Turnhout, Brepols, 1969, p. 181 : «Ce nom de "personne" se prend donc de trois ou quatre façons - et peut-être davantage: d'une certaine façon par les théologiens, d'une autre par les grammairiens, et d'une autre encore par les rhéteurs, ou dans les comédies, ainsi qu'on l'a montré plus haut » (nous traduisons). 
fondements comme dans son sens ultime, la persona est une notion théologique, au service d'une exégèse du mystère trinitaire - et c'est la théologie, du reste, telle qu'Abélard la conçoit (à savoir plus comme une rencontre entre le logos et le Mystère que comme un discours sur Dieu) qui enveloppe toute sa pensée, jusqu'à déclencher les polémiques intellectuelles dont il fera l'objet.

C'est de là, donc, que nous partirons dans cet article pour tâcher d'élucider chez lui cette déclinaison de la «personne». Nous en partirons comme d'un point focal à partir duquel nous irons chercher les autres acceptions (sans que cela dénie aux autres emplois une consistance propre). Notre but, par conséquent, sera à la fois d'examiner ce que chacun de ces emplois de persona signifie et de les situer dans la philosophie abélardienne tout entière : une philosophie, écrit J. Jolivet, dans le langage, et non pas $d u$ langage, c'est-à-dire une pensée qui certes débute et se développe dans et par le langage, mais sans cesser de viser - pour y trouver son sens - une fin qui le dépasse, à savoir la morale, et plus précisément : une théologie morale à forte teneur intellectuelle ${ }^{2}$.

\section{La question de la Personne Trinitaire : personne théologique et langage}

« Or il advint que je m'occupai d'abord de traiter du fondement même de notre foi en usant des similitudes de la raison humaine, et que je composais un traité de théologie sur l'Unité et la Trinité divines, à l'intention de mes étudiants. Ceux-ci exigeaient des raisons humaines et philosophiques, et réclamaient sans cesse ce qui peut se comprendre, de préférence à ce qui peut se dire : ils disaient en effet qu'un énoncé verbal que la compréhension ne suivrait pas, serait inutile, qu'on ne peut croire ce qu'on n'a pas compris au préalable, et qu'on se rendrait ridicule à prêcher aux autres ce qu'on ne pourrait saisir par l'entendement, pas plus que ceux qu'on enseignerait ; le Seigneur luimême le prouve : des aveugles guideraient des aveugles $»^{3}$.

Dans son Historia Calamitatum, Abélard décrit le contexte dans lequel la Theologia Summi Boni (ou Tractatus) fut écrite. Il y raconte comment, sollicité par ses élèves, et animé par sa propre conviction de chrétien et de logicien, il décida de consacrer ses études et ses cours à la compréhension du mystère trinitaire traversé par la notion de persona. Son Tractatus commence par les questions suivantes : que signifie la distinction de trois Personnes dans une nature toujours identique à elle-même, et comment

2. J. Jolivet, Arts du Langage et Théologie, Vrin, Paris, 2000, p. 10.

3. P. Abélard, Historia Calamitatum (= HC), IX, in Id., Historia Calamitatum, éd. Monfrin, Paris, 1978, p. 82. 
concilier l'unité de nature de la Divinité avec la triplicité des Personnes ? ${ }^{4}$ Autrement dit, comment l'indivisible peut-il être multiple, et quelle est la nature de cette multiplicité ?

Ces questions, dans leur formulation même, dévoilent deux aspects fondamentaux de la pensée abélardienne : premièrement, une adhésion totale aux dogmes chrétiens pris comme des Vérités immaculées ; deuxièmement, le biais par lequel il s'en approchera. En posant la question de la signification de la distinction des Personnes, le philosophe entre à la fois dans le champ de la sémantique (signification) et dans celui de l'analytique (distinction) - ce qui préfigure la grammaire spéculative, la question de la Personne trinitaire devenant nécessairement une question théo-logique ${ }^{5}$.

Lisons son argument : « un sceau de bronze ; il est à la fois un et triple : il est bronze selon la matière et sceau selon la forme; le sceau procède du bronze et non l'inverse, comme le fils procède du Père et non l'inverse ; enfin, dans son action de sceller, le sceau scellant, tel l'Esprit Saint procédant du Père et du Fils, procède à la fois de la matière et de la forme, du bronze et du sceau en tant qu'instrument de scellement $»^{6}$.

Abélard explique ici par analogie ou similitudo (comme il l'avait avoué à ses élèves : «je m'occupai d'abord de traiter du fondement même de notre foi en usant des similitudes de la raison humaine ») les fondements de sa théorie des Personnes. Le sceau de bronze peut se concevoir sous différents rapports : bien qu'ayant une unité essentielle, l'objet «sceau de bronze» peut se penser selon sa forme, selon sa matière, et selon une matière formée pour quelque chose. Dans le même temps, un autre type de rapport a lieu, «le fait de procéder»: la matière est formalisée et la matière formalisée vient de la jonction de la matière et de la forme (jonction conçue comme un

4. P. Abélard, Theologia Summi Boni $(=$ TSB), I, 1, in Petri Abaelardi Opera Theologica, vol. III, éd. E. Buytaert et C. Mews, Turnhout, Brepols, 1987, p. 86.

5. La logique ou discipline dialectique, le bon usage de la raison ou la distinction du vrai et de l'apparence, est la discipline qui nous permet de comprendre la Vérité : ainsi, la Personne du théologien nous renvoie nécessairement à la personne du dialecticien. La logique est le moyen dont nous disposons pour accéder aux mystères, voire ce qui nous ouvre à la théorisation de la question des Personnes trinitaires. La dialectique est l'outil nécessaire, une certaine condition de possibilité d'une pensée sur la Personne théologique. Non pas pour démontrer, mais pour défendre la foi, dirait Abélard ; non pas pour la connaître, mais pour la comprendre. Abélard, dans son Invective contre quelqu'un qui n'entendait rien à la dialectique, qui pourtant trouvait mauvais qu'on l'étudiât, et pensait que ses enseignements n'étaient que sophismes et tromperies (= Lettre XIII, in J. Jolivet, Abélard ou la Philosophie dans le Langage, Paris, Cerf, 1994, p. 150), dira : «sans elle <la dialectique>, il est impossible de résister aux attaques des hérétiques et des infidèles et de leur fermer la bouche en leur opposant de bonnes raisons »; l'homme, incapable de répondre aux attaques contre la foi au moyen du miracle, doit le faire par la parole dialectique. "Je crois, écrit Abélard, que nul ne saurait comprendre pleinement ces choses à moins de passer ses nuits à étudier la philosophie et avant tout, la dialectique » (ibid., p. 152). Pour finir, notons ceci : «Christ signifie le logos, chrétiens doit vouloir dire logiciens, et donc la doctrine chrétienne, logique » (ibid., p. 154).

6. P. Abélard, Theologia Scholarium (=TS), III, in Petri Abaelardi Opera Theologica, vol. III, p. 143. 
troisième mode de Dieu et non pas comme une simple addition des deux autres). Dans le cas de Dieu, ou de l'Un, à savoir la substance divine, l'enjeu et sa solution sont identiques. Il ne pourrait en être autrement : l'Un étant simple et indivisible, et tout autre qu'un individu, aucune division réelle ne peut y apparaître (puisque pour Abélard il n'y a rien de réel que l'individu et dans l'individu, l'individuel). Les trois Personnes ne peuvent donc absolument pas être réellement, ou individuellement, distinctes, elles ne sont pas des choses, des res. La modalité, par conséquent, à savoir la conception de cette unité « selon quelque chose », ou la différenciation selon un rapport, est la seule à nous permettre de penser une Unité multiple. Les modes, en tant que « raisons d'être différentes », " ayant des propriétés distinctes », respectent le caractère multiple de l'unité.

La différenciation dans l'unité est donc une chose tout autre que sa division. Différencier implique une «modalité », des approches du «même », tandis que la division ferait de l'unité une triplicité (ou même, une réification). Ainsi, Abélard, en décrivant la perfection du Bien suprême, à savoir Dieu (Dieu comme complètement achevé ou par-fait) explique que la Sagesse même de Dieu incarnée (le Christ ou le logos) ne l'a pas divisé, mais l'a différencié au moyen des trois noms quand, pour trois raisons, elle l'a nommé Père, Fils et Esprit Saint. En ces trois caractères : la puissance, la sagesse et la bonté consiste donc l'entière perfection du bien, et aucun d'eux ne vaut sans les deux autres. Autrement dit, le rapport entre les Personnes est d'un ordre nécessaire : quelqu'un de puissant, mais qui ne sait régler ce qu'il peut selon la loi de la raison, n'a qu'une puissance funeste et pernicieuse ; s'il est sage et s'il agit avec discernement, mais qu'il est impuissant, il n'aboutit à rien; et s'il est puissant et sage sans être aucunement bon, il est d'autant plus porté à nuire que sa puissance et son habilité lui permettent d'accomplir plus sûrement ce qu'il veut : celui que ne meut aucun sentiment de bonté ne laisse rien espérer aux autres de ses bienfaits ${ }^{7}$.

Par conséquent, lorsqu'Abélard dit que la différence ne suppose pas nécessairement une divisibilité, et qu'il s'agit ici d'une distinction nominale, il n'est pas seulement en train d'expliquer comment on pourrait résoudre ce (faux) problème de l'unité trine, mais il réfute aussi les réalistes, à savoir ceux qui, en réifiant les Personnes, c'est-à-dire en faisant d'elles trois essences - en les individualisant -, contredisent la Sacra Pagina. Abélard, sur ce point, insistera sans cesse pour dire qu'il n'y a qu'une seule essence, ou bien que la substance des Personnes divines est la même selon l'essence, et qu'elles ne sont diverses que comme des choses diverses par définition. Le propre du Père est le pouvoir; il est distinct du propre du Fils qui est de connaître, et du propre de l'Esprit qui est d'être bon ${ }^{8}$.

Chaque Personne est, sous une certaine modalité (i.e. en signifiant des choses distinctes), Dieu sans que cela nous conduise d'un côté à une

7. P. Abélard, TSB, p. 25.

8. Voir J. Jolivet, La Théologie d'Abélard, Paris, Les éditions du Cerf, 1997, p. 37. 
équivalence exclusive des termes ou, d'un autre côté, à une triple essence. Ainsi, le Père seul est Puissance, le Fils seul est Sagesse et l'Esprit Saint seul est Bonté, mais les trois se rencontrent entièrement en Dieu?. Souvenonsnous que leur rapport est nécessaire. Le philosophe veut plus énoncer les attributs propres à chaque Personne que les individualiser - ce qui serait absurde, et d'ailleurs hérétique.

Ainsi, puisqu'une substance simple n'a pas de parties, c'est-à-dire n'admet pas l'existence en elle d'entités réellement distinctes, les Personnes ne sauraient être réelles. Les Personnes sont plutôt des «voies»- des « outils»-qui nous permettent de nommer l'Unité de trois manières sans pour cela la diviser. Car Dieu se dit de trois manières : Père, Fils et Esprit Saint.

La Personne trinitaire n'est donc «rien d'autre » qu'un nom, car les noms n'ont pour objet que de signifier. Reformulons : la « Personne» est un nom, un agent significatif par lequel nous produisons des intellections : c'est ce par quoi Dieu se rend «connaissable » pour nous. La Personne trinitaire, en tant que nom, est donc l'outil - le plus humain des outils - permettant d'exprimer quelque chose de propre à chacune des Personnes en Dieu.

\section{La Personne Grammaticale : Grammaire et Dialectique}

Suivant la ligne que la Personne théologique (en tant que nom) a tracée, cette deuxième partie d'ordre linguistique a pour but d'exposer la théorie abélardienne du nom et ce qu'on appelle son «nominalisme »- ou plutôt son «non-réalisme», pour parler comme J. Jolivet (que nous suivons) -, cela afin de comprendre comment, pour le grammairien, le nom se rapporte à la personne. Il s'agira donc ici d'articuler le nom, la signification et la personne.

Bien que les débats concernant Abélard et la querelle des universaux soient féconds et fondamentaux pour comprendre son œuvre, nous consacrer à cette entreprise outrepasserait les limites de cet article. C'est donc uniquement dans le cadre de la notion de «personne » que l'on considère ici l'universel. Or, la personne grammaticale ne vient pas seule ; elle s'inscrit pour ainsi dire dans un «paysage », celui de la réflexion sur «le nom », sur les normes linguistiques permettant de l'utiliser et sur l'universel. C'est dans l'espace couvert par cette articulation que se place l'analyse qui suit.

Un « vocaliste», un «nominaliste », un « conceptualiste», un «nonréaliste » : plusieurs « étiquettes » ont servi à caractériser Abélard au cours de l'histoire, chacune dotée d'une certaine justesse. Les débuts vocalistes

9. Nous profitons du terme « rencontre » (conueniunt) présent et fondamental à la notion de status (centre de la théorie des universaux abélardienne), notion qui permet de penser la possibilité d'une diversité non individuelle au sein du «même». 
d'Abélard, le passage, d'importance cruciale, à la distinction entre vox et sermo, et l'interprétation de cette distinction d'après la notion de «concept » comme présence mentale (cette dernière, étant, peut-être, la plus risquée des théories proposées), tout cela justifie partiellement l'une ou l'autre des désignations qu'on a rappelées. Toutefois, comme on l'a dit, celle du nonréalisme nous semble convenir le plus à la pensée abélardienne - une pensée multiple et changeante, mais qui, dans ses fluctuations, n'a jamais abandonné « le nom ». Ce «nom», chez Abélard, est à la fois le socle d'une réponse aux débats de l'époque et le fondement de sa propre doctrine. À cet égard, s'agissant du « nom », la théorie abélardienne des universaux est aussi bien réactive qu'autonome.

C'est sous le patronage, parfois conflictuel, des maîtres nominalistes, en opposition à la thèse réaliste d'une réalité de « ce qui est capable d'être prédiqué de plusieurs » (quod de pluribus natum est aptum praedicari), pour tout dire d'une réalité de l'universel, que se place d'abord la pensée d'Abélard. Ce dernier, tantôt proche, tantôt éloigné de Roscelin de Compiègne, peut être justement désigné, on l'a dit, comme non-réaliste, et non comme nominaliste radical. Voyons pourquoi.

Qu'est-ce que l'homme? L'homme est un universel, à la différence de Socrate, qui est un singulier (quod de uno solo praedicatur). Dès l'origine, il en va ainsi : dès sa première formulation, en effet, c'est-à-dire dès son impositio (la découverte d'une façon de grouper dans la nature des choses ${ }^{10}$ ), l'universel est un universel et le singulier, un singulier. À la différence de Porphyre, Abélard définit l'universel comme ce qui est capable d'être prédiqué, et non pas comme ce qui l'est effectivement ; pour être universel ou singulier, le nom n'a pas besoin d'une prédication actuelle. Cette capacité de prédication nous renvoie ainsi à un certain état des choses (rei status) et non pas à la chose individuelle actuelle dont il est question. Cela confère à la pensée abélardienne une allure formelle, dès lors qu'on considère davantage la manière de parler des choses que les choses elles-mêmes. Ainsi, la nominatio est la relation d'un nom à une chose dont il est le nom, et l'impositio (ou inventio) est l'établissement d'un nom ou l'acte par lequel le nom devient le nom de quelque chose. De manière très large, pour Abélard, l'universel est un mot et on l'attribue à un sujet en vertu d'un certain "mode d'être de ce sujet», et non pas, tout bonnement, en vertu de «ce sujet». L'être-homme, différent de l'homme, n'est point une chose, et l'on pourrait même dire qu'à strictement parler, il n'est rien. "Toute cause d'imposition d'un nom universel est en elle-même infinie et n'est incluse en aucune limite de choses », écrit Abélard dans sa Logique ${ }^{11}$.

10. Cf. P. Abélard, Logica Ingredientibus (=LI), 20 in M. de Gandillac, Euvres choisies d'Abélard, éd. Montaigne, Paris, 1945, p. 108.

11. P. Abélard, LI, 85 in B. Geyer (éd.), Peter Abaelards philosophische Schriften, Münster, 1919, p. 100 (Beiträge zur Geschichte des Philosophie und Theologie des Mittelalters, 21/1-3) : «[...] omnis causa impositionis universalis nominis in se ipsa infinita est nec ullo rerum termino inclusa »; et plus loin: «Sicut enim rationale infinitam naturaliter 
D'un côté les choses, de l'autre les idées; le palletien, combattant le nominalisme avec le réalisme et le réalisme avec le nominalisme, au moyen de la question «qu'est-ce qu'un mot? », paraît adopter sur le problème des universaux une position de type «ni-ni» et non pas «soit-soit». L'universel n'est ni une chose ni une idée, mais un mot, et comme il l'explique dans ses écrits logiques ${ }^{12}$, outre la chose et l'intellection, apparaît ce tiers qu'est la signification des mots.

La réponse d'Abélard surgit donc d'un nouvel espace, d'une nouvelle origine qui place au centre du problème des notions qui n'avaient été déterminantes $n i$ dans le réalisme $n i$ dans le nominalisme, à savoir : le sermo, la significatio, le dictum et le status. Autrement dit, tout en répondant au même questionnaire de Porphyre, les problèmes abélardiens vont se poser autrement et se trouveront animés par d'autres figures. Raison suffisante pour que cette théorie prenne un autre nom, un nom propre, aussi complexe, et même confus, que ce qu'elle exprime (ainsi que J. Jolivet lui-même le dit) : le non-réalisme.

Mais revenons à la personne grammaticale en reprenant notre point de départ : la Personne théologique, disions-nous, est un nom, et, ajoutons-le, un nom signifie.

Parler de grammaire nous situe dans le domaine des normes dans la composition d'une phrase, au niveau de la congruence. Cette discipline, pour Abélard, vient nécessairement avant la correcte attribution des prédicats (la dialectique), et constitue donc une première partie (temporellement, mais seconde en dignité) dans cette institution humaine qu'est le langage. Cependant, le philosophe aura tendance à s'installer d'emblée dans le langage, c'est-à-dire qu'il n'aura pas de mal à traiter de manière logique des faits grammaticaux ; la frontière entre grammaire et dialectique n'est ni lisse ni fixe. Or, bien que le champ de la congruence ne soit pas le même que le champ de la vérité (ce qui nous permet de concevoir une bonne construction avec une mauvaise prédication: "l'homme est une pierre»), chaque discipline, tout en gardant son autonomie, participera à ce que J. Jolivet, de nouveau, appelle « une discipline unifiée du langage ».

Le langage pour Abélard, nous l'avons dit, est porteur de la Vérité, de la Révélation, et c'est à travers lui, du reste, que les pensées relatives aux choses se sont exprimées et ont été comprises. Cette compréhension a pour base une intellection, et « derrière » elle, un mot.

Le langage est donc porteur de deux faces : il tient à la fois aux choses et à la pensée, et il a pour fonction de transmettre en utilisant le mot comme outil des diverses médiations ${ }^{13}$. Les mots, noms et verbes, ont une double

impositionis causam habet, ita et rationalitas et quodlibet universale ». Donc «Qui enim hoc nomen imposuit rebus, scilicet homo [...], sicut numerum definitum habebat, ita nec omnem causam quae est esse hominem certa intelligentiae meta comprehendit ».

12. Cf. P. Abélard, LI, I, 16, in Gandillac éd. citée, p. 102.

13. Cf. J. Jolivet, Arts du langage et théologie chez Abélard, p. 172. 
couche ; l'une concernant les choses, et l'autre, les intellections. En effet, les mots signifient les choses en constituant une intellection, en désignant à un auditeur l'intellection de celui qui parle et en produisant en lui une intellection semblable. Dès lors, si un mot, compris comme «émission ou son », signifie, c'est qu'en lui quelque chose se surajoute à son être physique, ce quelque chose étant sa fonction significative. Abélard distingue ainsi un aspect matériel et un autre aspect tenant à la signification du mot ; materia vocis et vox significativa ou sermo. Vox (distinguée du sermo) passe du côté de la chose en se plaçant dans la sphère naturelle, tandis que le sermo, comme signifiant ou comme fonction significative du mot, dépend d'une institution, d'une convention humaine ${ }^{14}$.

Dans cette théorie, large et complexe, nous nous intéressons à la signification comme phénomène engageant deux individus, c'est-à-dire comme phénomène interpersonnel. Chez Abélard, signifier veut dire « engendrer une intellection semblable dans l'esprit de l'auditeur». Cela veut dire concevoir et engendrer non pas un mot ni une chose, mais une intellection au moyen du mot. Signifier apparaît ainsi comme quelque chose de fondamental et de propre tant au langage qu'aux agents (sujets de l'action) qui participent à cet acte de signification, car le mot est un mot dirigé vers quelqu'un. Ces deux participants sont, d'une part, celui qui nomme et qui exprime, et d'autre part, celui qui reçoit et qui engendre aucun des deux ne se caractérisant par une pure activité ou une pure passivité. La signification est un phénomène de couches; la signification suppose un rapport entre un « je », un «tu » et un «objet/il». Cela constitue une première approche des personnes grammaticales.

Or, cet engendrement (dans l'esprit de l'autre) ne vient pas seul. Il est l'aboutissement d'un processus cognitif intellectuel qui commence dans le sujet de l'émission. Processus permettant l'imposition d'un nom et qui se déroule de la manière suivante (nous nous appuyons sur le De Intellectibus) : au fondement de toute connaissance, il y a la sensation qui «touche légèrement » l'objet, puis vient l'imagination qui, en se débarrassant d'une extériorité, saisit la chose de manière confuse ou indéterminée - car imaginer, c'est accueillir simplement la chose sans considérer encore en elle ni nature ni propriété. Enfin vient l'intellection. Celle-ci, ayant comme acte essentiel celui de l' «attentio », débarrassée de toute extériorité, observant à travers les yeux de l'esprit, intellige « sa chose ». Par les images, l'intellect vise une nature ou propriété de la chose en se plaçant devant son objet ; et il se l'approprie. De la sensation à l'intellection, en passant par l'image, un processus d'abstraction permettant la fondation des intellections, comme portée au terme de la connaissance, a lieu. C'est ainsi que nous obtenons les «connaissances des natures qui fonderont l'imposition des noms, l'attribution du prédicat au sujet et enfin le raisonnement ${ }^{15}$. Autrement dit :

14. Bien qu'il y ait une identité d'existence (in essentia identitas) - vox et sermo se référant à la même chose -, leur origine (ou nativitas) est différente.

15. J. Jolivet, Abélard ou la Philosophie dans le langage, p. 66. 
c'est parce que l'on vise (on délibère), qu'on «connaît », et que l'on peut ainsi nommer pour pouvoir enfin communiquer.

Mais, qu'en est-il de la personne?

L'attentio, viser une nature ou une propriété, se livrer à une chose comme acte de l'âme - ne pourrait pas ne pas être un phénomène d'ordre personnel, c'est-à-dire un phénomène propre et exclusif à un "point de vue particulier », car le sujet - le sujet mettant en œuvre cette puissance - dans cet acte, se fait ; d'une certaine manière, la personne de l'acte intellectif, en se posant devant son objet, s'impose comme son propriétaire, voire comme l'auteur de la chose concernée. Ce n'est pas l'homme en général qui vise ; toute visée n'est jamais que la visée de quelqu'un. La chose nommée, inscrite maintenant dans le monde langagier (c'est une chose dite), se détermine. Le langage étant une institution humaine, la chose s'humanise. La visée comme condition de possibilité de l'intellection est traversée par ce que la phénoménologie appellera plus tard, une intentionnalité, la chose, la pensée de la chose, cette chose-là, n'étant telle que pour celui qui la saisit. C'est pour cette même raison que l'engendrement de l'intellection dans l'esprit de l'autre (c'est-à-dire : signifier) ne peut qu'être semblable, et non pas de l'ordre du «même». Reformulons notre phrase, par conséquent: viser, s'intéresser ou faire attention à une chose en tant qu'elle est telle chose, ne pourrait pas ne pas être un phénomène d'ordre personnel (de propre à un « singulier »).

La signification, essentielle à la communication, se voit affectée d'une double contrainte : une cause et une similitude. Une cause, car il n'y a pas de pensée sans objet; penser, c'est toujours penser quelque chose ; et une similitude, car il faut poser qu'entre mon intellection et celle de l'autre, bien qu'elles soient nécessairement liées par la même «cause », dans l'acte de transfert, une coïncidence absolue n' aura jamais lieu.

De cela, de la présence d'un émetteur, d'un récepteur, et de l'objet de la transmission, c'est-à-dire en considérant les sujets participant à la signification, et la distance qui les sépare, nous pouvons tirer une première analogie avec la réflexion trinitaire : quoiqu'unifiés, et participant d'un seul et même acte (la significatio), autre est le propre de celui qui parle (celui qui impose le nom - la forme), autre le propre de celui à qui l'on parle (celui qui engendre l'intellection à partir de ce nom reçu - la matière), et autre, enfin, le propre de ce dont on parle (le nom et la chose - la matière formée). Ces trois dimensions du même sont exprimées dans les trois «personnes grammaticales » : le « je », celui qui vise et qui impose, le «il », cette chose visée et nommée appartenant autant à l'émetteur qu'au récepteur, et le «tu », celui qui l'engendre.

Abélard exposera une analogie encore plus directe entre Personnes trinitaires et personnes grammaticales dans sa Theologia Christiana. Il dira qu'on peut bien admettre qu'un même homme soit trois personnes : celui qui parle, celui à qui l'on parle, celui dont on parle, la substance des trois 
étant la même, et les personnes ne pouvant être distinguées que selon leurs «propres », exprimés par leurs définitions ${ }^{16}$.

La personne a ainsi un double rapport au «personnel»: la personne s'exprime par ce qui lui est propre, ce que son nom dit. Et la personne est un nom, un outil, sous la forme d'un rapport. Or, l'importance de la signification et du sermo, comme fonction significative du mot, est radicale pour concevoir un universel, ce "prédicat de plusieurs", ou bien cette «multiplicité une». L'universel doit être le prédicat d'un sens et non pas uniquement d'une émission. Car la vox ne peut pas avoir d'universalité, elle est une chose, elle est uniquement une chose (répétons cela : il n'y a de réel que l'individu, et dans l'individu, l'individuel). Le sermo comme sens permet ainsi une pluralité (modale) dans l'unité d'un mot. Toujours dans la Theologia Christiana, Abélard écrit : «Le nom Dieu devrait, certainement, être un universel, car plusieurs choses sont distinguées personnellement en lui ${ }^{17}$. Autrement dit, dans l'universel et en Dieu, certaines choses sont distinguées personnellement et non pas individuellement. Les personnes grammaticales (le «je, tu, il»), toutes distinctes des «choses», et les Personnes trinitaires participant d'une seule et même essence, doivent se concevoir, nous y insistons, sous une forme de diversité, de différence et non pas comme une simple unité ; l'unité-multiple ne peut qu'être conçue sous la forme d'une personnification. Ainsi, c'est uniquement en suivant cette approche de l'universel (de l'«homme » comme prédicat de plusieurs et de Dieu comme Un et trine), que le multiple ne se réifie pas.

Avec la notion de sermo nous trouvons un autre terme également décisif pour réfléchir à la multiplicité dans une unité indivisible : le status.

« Les hommes singuliers diffèrent les uns des autres, mais tout en différant aussi bien par leurs essences que par les formes qui leur sont propres [...] ils se rencontrent en ce qu'ils sont des hommes. Je ne dis pas qu'ils se rencontrent dans l'homme car l'homme n'est aucune chose sinon une chose individuelle, mais dans l'être-homme (esse hominem) qui n'est ni l'homme, ni aucune chose $»^{18}$.

La rencontre des hommes se fait dans le statut d'homme, c'est-à-dire dans son expression grammaticale par une proposition infinitive: l'esse hominem. Un état de l'homme (et non pas «l'homme») qui exprime, à la fois, un homme impersonnel (au sens individuel) mais personnifié, avec une puissance multiple - comme nous l'avons déjà vu pour les personnes grammaticales. Le status nous permet de concevoir une multiplicité intérieure (discretio) sans pour cela diviser l'unité ${ }^{19}$.

16. Voir P. Abélard, TC, III, p. 175.

17. Ibid., p. 171.

18. Ibid., p. 19.

19. Dire qu'une chose est séparée d'autres choses ne revient pas à dire qu'elle diffère de ces autres choses, car toute chose, par définition, est séparée. L'unité de la chose signifie ainsi le 
Une chose est certaine, la notion de personne se complexifie. La personne est un nom ; la personne a quelque chose de propre et de partagé ; la personne, sans jamais abandonner une seule et même essence, est susceptible d'être conçue sous divers modes; la personne n'est pas «à » un individu, la personne est un rapport. Une clarification est-elle possible ? Quand le terme "personne» est utilisé pour évoquer une réalité concrète, une existentia, ne devrions-nous pas plutôt penser au terme «individu»? Nous faut-il distinguer la notion de «personne» de celle, sous forme adjectivale, de «personnel » («personne » étant la figure par laquelle une distinction, et non pas une individualité, est pensée, et «personnel » ce qui est propre à cette figure) ? À moins qu'on ne doive accepter une confusion dans la notion abélardienne de personne ${ }^{20}$ ? C'est cette troisième hypothèse que nous retenons : la «personne » nous semble être chez Abélard un terme complexe, composé. Insistons pour conclure cette deuxième partie, en abordant brièvement la question du dictum.

Une fois suivies les normes grammaticales, et une fois la phrase construite de manière congruente, la question de la vérité (la question dialectique) s'impose. Ici, la «personne grammaticale » (le sujet du prédicat) pose un problème d'ordre existentiel. Le «je, tu, il», les personnes grammaticales, en tant qu'elles sont liées in existentia aux prédicats, empêchent un rapport nécessaire de vérité ou de fausseté de la proposition, et donc, empêchent sa validité. Il semble que l'attribution d'un verbe à une personne, dans sa particularité, soit conçue par Abélard comme un phénomène proche d'une individualité (une réalité), voire identique à elle. Cette individualité, cependant, n'est pas nommée comme telle par le philosophe, mais elle paraît sous l'idée d'«un mode personnel » de l'attachement du verbe au sujet. Nous savons qu'à la rigueur, pour qu'une phrase soit dite vraie ou fausse, le prédicat ne doit pas s'attacher à la personne qui fait le verbe (exemple: Socrate currit) mais bien à l'intellection que cette proposition engendre (Socratem currere). Cette tournure à l'infinitif exprimant «ce que la proposition dit» ou le dictum propositionis. En d'autres termes, pour qu'une proposition logique ait lieu, il faut effacer l'existentia de la personne ${ }^{21}$.

fait d'être séparée (question essentielle à toute substance existante). L'unité est essentielle à la chose sans être genre ni différence, et « les individus consistent seulement dans la séparation personnelle », c'est-à-dire une séparation existentielle (réelle). Cf. J. Jolivet, Arts du Langage et Théologie, p. 103.

20. Confusion, d'ailleurs, déjà présente dans la Personne trinitaire, ainsi - selon nous - que dans le traitement abélardien de la question du dictum propositionis.

21. P. Abélard, Glossae super Peri Hermeneiasi, XII, 5-10, in Adam Balsamiensis Parvipontani, Twelfth Century Logic, Texts and Studies, Abaelardiana inedita: 1. Super Periermenias XII-XIV, Sententie Secundum, Ed. Storia e Letteratura, 1958, p. 97. Socrates currit necessario (Socrate court nécessairement) est une proposition purement factuelle (puro inesse), d'où se tire la proposition «il est nécessaire que Socrate court» (necesse est Socratem currere), proposition proprement modale. Cette proposition peut se prendre de re (de manière existentielle, en séparant la phrase en «sujet» et «prédicat de ce sujet») ou de sensu (en conçevant la proposition à l'infinitif, c'est-à-dire, en la comprenant comme une 
Insistons sur ce point: le traitement de la personne en grammaire n'est pas univoque. Parfois, Abélard tend à la concevoir comme un agent significatif, comme un nom, notamment en la liant à la question de la signification et de son rapport à l'universel. D'autres fois, elle est plus proche d'une existentia. Quoi qu'il en soit, il nous semble que lorsqu'elle est pensée de façon matérielle (impliquée matériellement), c'est en termes d'individu plutôt que de personne qu'elle doit être conçue, dans la mesure où la «personne » est fondamentalement un nom (et comme tel, elle se rapporte aux choses aussi bien qu'à la pensée), tandis que l'existentia de cette personne suppose précisément un attachement « aux choses ».

C'est Abélard lui-même qui suggère cette conclusion. En observant la terminologie abélardienne, en effet, nous constatons que la notion d'individu reste quasi exclusivement logique, tandis que celle de personne s'inscrit, elle, dans un cadre théologique. Tout cela est clair, sauf en un cas : le dictum. Ici, il nous semble que si Abélard recourt à l'expression «personne grammaticale » pour exposer le conflit de l'attachement existentiel en grammaire et en logique, ce n'est pas pour réfléchir au terme de «personne». Son emploi lexical procède simplement d'une convention terminologique. Dans le cadre du dictum, par conséquent, penser la «personne grammaticale» comme «individu grammatical» semblerait préférable, l'effacement de la personne se comprenant plutôt comme une «neutralité existentielle».

Passons maintenant à la rhétorique, dernière partie de cette étude.

\section{La personne Rhétorique}

Un constat, pour commencer. Non seulement nous ne conservons pas la Rhétorique d'Abélard, mais il est clair que cette discipline n'est pas aussi présente chez lui que la dialectique - bien qu'ils soient conçus comme quelque chose d'organique et d'unitaire, les arts du langage présentent en effet une certaine hiérarchie, une structure - on l'a dit - par enrobement. Cela, toutefois, ne signifie pas que la rhétorique, comme domaine, comme objet, soit complètement absente de la pensée abélardienne, ni que, par conséquent, on ne puisse tirer de son œuvre une certaine doctrine relative à cette discipline. En tant que partie du trivium, elle occupe certainement une place chez Abélard, et c'est elle, dans son rapport à la notion de personne, que nous allons tâcher d'explorer.

$\mathrm{Si}$ la dialectique traite des arguments et la grammaire de leur construction, que reste-t-il à la rhétorique ? Compte tenu de notre analyse, quelle est $s a$ personne ? Celle-ci se rapporte-t-elle à celle du comédien ?

unité). La proposition modale devient ainsi impersonnelle (enuntiatio impersonalis) : on ne peut pas rapporter la force de l'énonciation (vim enuntiationis) à la personne, mais à ce que dit la proposition. 
Essayons d'abord de situer la rhétorique de manière générale. Dans l'image du Jardin des Délices (Hortus Deliciarium) ${ }^{22}$, la rhétorique est représentée avec, dans une main, une tablette, et dans l'autre, un stylo. On y lit : «grâce à moi fier orateur, ton discours se fortifiera ». La rhétorique a un caractère amplificateur de fortification du discours. Quelque chose se surajoute au contenu «objectif» de ce qui est en train d'être communiqué, quelque chose qui amplifie, qui illumine, qui «met de la couleur » ou vivifie les mots afin de les rendre plus puissants. Voilà le champ de la rhétorique.

Cette idée sur la dimension «stylistique» du discours, nous la trouvons sous la plume du Maître Pierre dans le chapitre III de l'Historia Calamitatum. Lisons :

«J'allai donc entendre ce vénérable vieillard <Anselme>. C'était à la routine, il est vrai, plutôt qu'à l'intelligence et à la mémoire qu'il devait sa réputation. Allait on frapper à sa porte et le consulter sur une question douteuse, on en revenait avec plus de doutes. Admirable aux yeux d'un auditoire, dans une entrevue de consultation il était nul. Il avait une merveilleuse facilité de parole, mais le fond était sans valeur et manquait de sens. Lorsqu'il allumait un feu, il remplissait la maison de fumée, mais ne l'éclairait pas. C'était un arbre tout en feuilles qui, de loin, présentait un aspect imposant : de près, et quand on l'examinait avec attention, on le trouvait stérile $»^{23}$.

Abélard fait part ici de la nullité de son expérience comme étudiant d'Anselme, maître de théologie à Laon. Ce dernier, porteur d'un discours sans aucun ingenium (aux yeux du palletien), était incapable d'illuminer et de stimuler sa classe. Anselme tenait un discours stérile et opaque «qui, en faisant du feu, remplissait la maison de fumée à la place de l'illuminer ». Comme si la possession de ses mots, de son discours, malgré sa facilité de parole n'était qu'apparente, et que n'était proposée là qu'une fausse source de connaissance, une simple répétition. Anselme, d'un côté, n'arrivait pas à répondre aux questions que lui-même produisait, et, de l'autre, paraissait n'être pas propriétaire de ses mots. Aux yeux du péripatéticien, la parole d'Anselme était immobile, noircie, plus portée par l'habitude que par un véritable acte de pensée. Bref, le théologien ne pensait pas, pas vraiment ${ }^{24}$.

L'ingéniosité se rapproche de ce qu'Abélard propose comme l'une des définitions de la dialectique - le bon usage de la raison -, mais sous un nouveau rapport, à savoir un point de vue plus proche de la forme du discours, de son aspect performatif. Si la raison dans sa bonne utilisation est

22. Hortus Deliciarum, encyclopédie manuscrite composée au XII ${ }^{\mathrm{e}}$ siècle par Herrade de Landsberg, abbesse du monastère de Hohenbourg (Sainte-Odile) en Alsace, et conservée à la bibliothèque de Strasbourg. Bibliothèque de l'École des chartes, 1840, tome 1, p. 238-261.

23. P. Abélard, HC, III, p. 149.

24. N'oublions pas qu'Abélard, par sa méthode dialectique - celle qui place en son centre la notion de quaestio - est l'un des grands précurseurs de la méthode scolastique à venir, basée sur la recherche et le mouvement de la pensée. 
la raison dialectique, la raison dans son bel usage - l'ingenium -, pourrait être pensée comme la raison rhétorique ${ }^{25}$. L'ingenium exprime une certaine puissance de la forme en tant qu'elle est liée à un contenu, et surtout, en tant qu'elle est l'objet d'une personne. Le penseur doit s'approprier sa parole, la particulariser, la personnaliser et, ce faisant, la rendre fertile.

Quelques lignes en avant, Abélard dira dans notre texte que l'opacité des leçons du maître de Laon le conduira à s'en absenter de manière régulière. N'était pas en cause le contenu des cours - il s'agissait d'études de théologie - mais le manque de créativité dans la parole du maître, son manque de personnalité.

L'usage de la notion de «personnalité » dans un discours, autrement dit la question de l' « auteur », pourrait sembler anachronique - ce scrupule tient probablement à ce qu'a imposé la modernité concernant la genèse, postmédiévale selon elle, du sujet pensant ${ }^{26}$. Cependant, il nous paraît fondé. Et pour justifier cette idée d'une puissance personnelle d'un discours, et souligner, ainsi, l'idée de "propriété » travaillée plus haut, rappelons brièvement quelques éléments relatifs à l'enseignement au XII ${ }^{\mathrm{e}}$ siècle.

L'éducation était alors payante, ce qui signifiait qu'un certain type de «marché des idées », une certaine compétition entre les maîtres avait naturellement lieu. Aussi les divers enseignants devaient-ils être capables d'intéresser leur classe potentielle ; un professeur devait, d'une manière ou d'une autre, séduire et se rendre visible aux élèves, non pas seulement au moyen de son objet d'étude, mais aussi par la forme de transmission de ce savoir, par son ingenium - un terme proche de ce que Giorgio Agamben, qui souligne l'idée d'engendrement, appelle genius ${ }^{27}$, et que Deleuze, quand il parle de la créativité, au sein du discours, nomme style ${ }^{28}$. Voilà qui pourrait

25. Soulignons le fait que cette beauté, dans le bel usage, n'est jamais complètement séparée de la bonté.

26. Sur ce point, nous suivons au contraire l' « archéologie du sujet » d'Alain de Libera, lequel replace le Moyen Âge au cœur de l'invention moderne du subiectum. Voir A. de Libera, Archéologie du sujet, vol. 2, Paris, Vrin, 2007, p. 397 ; voir aussi, Id., «Dénomination extrinsèque et "changement cambridgien". Éléments pour une archéologie médiévale de la subjectivité », in K. Emery $\mathrm{J}^{\mathrm{r}}$, R. L. Friedman, A. Speer (eds), Philosophy and Theology in the Long Middle Ages. A Tribute to Stephen Brown, Leiden, Brill, 2011, p. 451-470 ; Id., « Le direct et l'oblique : sur quelques aspects antiques et médiévaux de la théorie brentanienne des relatifs », in A. Reboul (éd.), Philosophical papers dedicated to Kevin Mulligan, Genève, 2011.

27. Voir G. Agamben, Profanations, Paris, Payot, 2006, p. 8 : «Genius. Les Latins nommaient Genius le dieu auquel chaque homme se trouve confié au moment de sa naissance. L'étymologie est transparente et reste encore visible dans la proximité de génie et d'engendrer. Que Genius ait eu quelque chose à voir avec engendrer apparaît d'ailleurs évident si l'on pense que pour les Latins l'objet "génial" par excellence était le lit : genialis lectus, parce que c'est là que s'accomplit l'acte de la génération ».

28. Voir G. Deleuze, C. Parnet, Dialogues, Paris, Flammarion, 2008. Dans les passages sur « $\mathrm{S} »$, Style, et «T $\mathrm{T}$, Tennis, dans la question de la création dans le jeu, la puissance du style comme quelque chose qui se surajoute et qui rend «génial» un joueur. Nous rapportons l'ingenium au style deleuzien (et au genius d'Agamben). 
ouvrir à une théorie de la rhétorique abélardienne, où le lien entre la forme et le contenu se ferait par l'ingenium, agent persuasif de personnalisation du discours.

Cela dit, revenons à la citation de départ en distinguant les deux dernières figures (la personne du rhéteur, celle du comédien) de celles du théologien et du grammairien. Un léger glissement y a lieu : alors que la personne du théologien et la personne du grammairien sont, de fait, l'un de leurs objets («personne théologique», «personne grammaticale»), distinctes du théologien et du grammairien comme sujets « agents » de leurs disciplines, la personne, pour le rhéteur, et la personne, pour le comédien, paraissent nous renvoyer non pas aux objets de ces arts, mais se référer plutôt aux sujets qui les exécutent. La personne, dans ces deux cas est le sujet de son action : la personne pour le rhéteur, c'est le rhéteur même ; et la personne pour le comédien, c'est le comédien.

Cette première distinction (objet d'étude et sujet agent de l'étude), si l'on tient compte des arguments qu'on a développés, nous permet de dévoiler d'ores et déjà notre thèse finale : le rhéteur et le comédien sont dans cette citation liés de manière intime. La thèse se trouve chez Abélard luimême, lequel, en insistant sur la question de la performativité et de la persuasion (dans le même passage de la Theologia Christiana), définira le comédien comme «un homme qui, à travers ses gestes, nous représente certains faits et certaines choses dites afin de produire un certain effet $\gg$. L'image est voisine de celle de l'orateur. Nous y reviendrons.

Persona, comme on sait, vient du grec prosopon, et veut dire, littéralement: ce qui «se présente à », ce qui vient «auprès de » (pros) la «vue» (ops); le masque de l'acteur, de l'hypocrite sort du chœur en opposant sa voix aux chants univoques, une voix singulière par le truchement de laquelle un destin particulier prend forme, qui modifiera la disposition des choses et d'autres personnages.

L'acteur vient incarner le destin d'un personnage auquel il prête sa voix à travers un masque ${ }^{29}$. Plus tard, dans le monde latin, le mot persona prendra la signification du masque de l'acteur, associée à l'idée de personare (étymologie attribuée à Boèce), qui veut dire résonner, retentir à travers, et désigne également le masque équipé d'un dispositif pour servir de portevoix.

L'acteur, dit Abélard, est la personne ; il est la personne en personnage. Celui qui représente des actions et des mots à travers des gestes. Celui qui apparaît devant un public et qui interprète un rôle au moyen d'un dispositif discursif afin de modifier, changer la route d'un personnage ou d'un récit. L'acteur, la persona, est un agent effectif et affectif. Retenons déjà les aspects suivants de cette figure: la performativité, l'expressivité, le fait d'affecter, et le masque (le dispositif).

29. Voir G. Gaillard, Rhétorique française à l'usage des jeunes demoiselles, Paris, Saugrain, 1787, p. 360. 
Dans les premières Gloses, on lit que la grammaire et la rhétorique sont au service de la dialectique, et Abélard ajoute que «pour faire des études en dialectique, il faut connaître, d'abord, au moins, l'essentiel de la rhétorique » car de l'unification entre la grammaire et la dialectique sortira ce que l'on entend par "grammaire spéculative», et les méthodes de preuve seront fournies par la dialectique et la rhétorique ${ }^{30}$. (Sans entrer dans le détail, acceptons que le domaine de l'inférence formelle ou dialectique, venant d'une relation parfaite et détachée de la nature, se distingue du domaine de l'inférence matérielle - rhétorique -, venant de la nature, de caractère imparfait). De cette distinction, Abélard dégage une définition de la rhétorique qui insiste plus sur son instrumentalité que sur la question de la vérité ou fausseté du discours qu'elle « forme » : en fait, la rhétorique existe principalement pour persuader, pour émouvoir (commovere) et pour produire (trahere) dans les dispositions (affectus) d'une personne un changement qui la fasse désirer ou rejeter une chose.

La rhétorique, comme discipline, porte sur le «non-nécessaire », elle se trouve exposée au contingent, s'occupant des manières, des apparences et non pas des « vrais » contenus des choses; elle répond aux questions : qui, quid, cur, quando, quomodo, quibus auxiliis. Pour soutenir cette idée, suivant le lecture de Boèce, Abélard expliquera qu'une chose est l'activité dialectique effective d'un dialogue réel que l'on codifie (un discours porteur d'une valeur logique), et qu'une autre est la question de la forme de ce discours, soulevée et inscrite dans le temps et limitée par un espace - par la nature. Autrement dit, les dimensions du contenu et de la forme sont différentes - ce qui ne veut pas dire qu'elles ne peuvent pas être liées ; quoi qu'il en soit, leur rapport n'est pas un rapport de nécessité. La rhétorique se trouve exposée à la contingence, en ayant pour objet (propre) les affections, les apparences et non pas la vérité.

Cette flexibilité du discours rhétorique, étranger à la vérité, cette perméabilité, cette plasticité, pourrions-nous dire, ne doit cependant pas être comprise - ni condamnée - comme une pure limitation. La rhétorique amène à différents outils linguistiques ayant une forte répercussion dans la pensée abélardienne, notamment celui de la translatio, figure majeure explicitant le statut significatif, institutionnel et subjectif du langage.

Translatio, «figure » ou «trope », désigne le fait de prendre un mot dans un sens qui n'est pas son sens propre, soit pour orner le discours, soit parce qu'on ne dispose pas d'un mot (propre) pour désigner la chose visée. Il s'agit d'un transfert dans lequel la figure s'oppose à la proprietas. Le sens de la phrase est ainsi compris dans une relative indépendance par rapport aux mots qui la constituent; elle « ne signifie ni par invention (imposition) ni par nature ${ }^{31}$, mais « autrement», elle signifie « indépendamment». Les tropes manifestent, c'est-à-dire rendent évidente, une troisième sphère : la sphère

30. Voir J. Jolivet, Arts du Langage et de Théologie, p. 19.

31. P. Abélard, LI, éd. B. Geyer, p. 84, 8-85, 16. 
de la pensée. Sphère située entre celle des mots et celle des choses, car une expression figurée ne peut être comprise qu'à la condition de ne pas croire que ce qu'il y a dans les mots transpose simplement et exactement ce qu'il $\mathrm{y}$ a dans les choses. Le trope ajoute une deuxième couche subjective dans l'usage des mots (outre la dimension humaine de l'institution), une couche personnelle, où intervient une appropriation, une croyance, une fiction. Autrement dit, du sein du langage, nous ne pouvons pas déboucher directement sur le monde, il faut d'abord traverser la sphère de l'esprit, de ses passiones, car dans l'usage des mots, et ici, en particulier, dans la translatio, une deliberatio, un acte de l'esprit, un jugement a lieu ; aussi bien dans l'élection personnelle du mot que dans l'acte d'ornementation - qui aboutit, encore une fois, à une similitude. Ce qui est en jeu, c'est l'intellection (et toutes les autres passiones) comprise comme mise en «perspective», comme mise en place nécessairement solidaire d'une conception particulière du monde. C'est en fait l'esprit du rhéteur qui se présente devant le récepteur de son discours, devant l'esprit de ce dernier. Le rhéteur se place devant son public afin de l'affecter. Nous voyons donc qu'ici le rapport du rhéteur avec le comédien, le pros-opon (« devant la vue »), est indiscutable. Tant l'acteur comme personnage, que le rhéteur comme «homme des affaires du monde » ou «substance rationnelle de la nature ${ }^{32}$ se trouvent 《dans la contingence » et ont pour but de commovere un autre à travers une certaine apparence, que ce soit sous la forme d'un personnage ou d'un dispositif. Notre thèse trouve son soutien.

De cette analyse, à partir de la question de la similitude et de la persuasion, nous pouvons déjà tirer quelques conclusions autour de la rhétorique et de ses outils. Premièrement, le trope témoigne à la fois de l'indigence du langage, de sa limite, de son imperfection - car c'est à travers lui qu'on essaie de combler un vide linguistique, un manque - et de ses ressources, de sa puissance et de ses possibilités - puisque c'est depuis et dans le langage que l'on trouvera le remède. Deuxièmement, la rhétorique tant dans l'usage du trope comme moyen de vivifier un discours afin d'affecter un auditoire, que comme moyen, en général, de persuader - se trouve très proche, au moins analogiquement, de la notion de significatio, car on doit se rappeler que signifier veut dire engendrer dans l'esprit de l'autre une intellection semblable, et non pas une chose. Dans le deux cas, un transfert, une inter-personnalité a lieu - intellectuelle dans l'acte de signifier, et affective dans le cas de la rhétorique/comédie.

Nous constatons ainsi que la rhétorique, que l'on pensait pouvoir définir comme outil «purifié » (et à la fois maudit) de tout contenu - comme une simple forme à appliquer -, nous apparaît comme l'ingenium en tant qu'elle est porteuse d'une dimension «poétique », créative et éthique, c'est-à-dire qu'elle apparaît liée à la personne et à son acte : au rhéteur. La capacité d'émouvoir, de commovere - de mettre en mouvement -, de changer la disposition du récepteur au moyen d'un outil (tout en acceptant qu'il s'agisse

32. P. Abélard, TC, III, p. 181. 
d'un outil non-nécessaire et mondain) rend cet art potentiellement noble. Il s'agit bien d'une potentialité, et non pas d'une noblesse assurée : dans les mains d'un anti-dialecticien - c'est-à-dire utilisée sans tenir compte du bon usage de la raison - cet outil ne peut que devenir nocif. Mise en acte, à l'inverse, par le dialecticien (par le rhéteur, cette persona qui est en même un dialecticien), elle atteint son plus haut degré et montre son visage le plus noble.

Or, dans ses travaux plus tardifs (précisément, dans les Commentaria in Epistolam Pauli ad Romanos), sans jamais abandonner l'idée de la nature « non nécessaire » (naturelle) de la rhétorique, Abélard, soucieux d'un bon usage des outils humains, dirigera cet art vers (et pour) la théologie. Ce sera elle qui fera le lien entre bonté et beauté, praxis et théorie, action (externe) et acte (interne - intentio) ; persona (dispositif) et personne (de l'acte). Le rhéteur devra donc connaître le bien, il devra adhérer à la volonté de Dieu afin de pouvoir se diriger vers lui. Et ce faisant, il se rendra capable d'affecter les autres (son auditoire, son lectorat) pour les inciter à faire de même. Le rhéteur devra faire de son masque ou de sa persona sa personne, c'est-à-dire qu'il devra ne plus être capable de séparer ce qui lui est propre de ce qu'il utilise comme moyen pour s'exercer. Le rhéteur, en somme, illustre, si l'on peut dire, une forme de «matérialisme théologique » : il est ce qu'il fait. Comme si le dispositif devenait personne en même temps que la personne dispositif, le rhéteur au service de la Vérité devient un instrument de Dieu.

Terminons cette troisième partie en développant, à partir de la notion de persona, l'idée d'une certaine profondeur de la surface dans l'art rhétorique abélardien et en soulignant une dernière fois la nature complexe de ce terme.

Dans les Commentaria, Abélard explique que «toute Écriture est un discours rhétorique qui n'a pas seulement un but pédagogique mais aussi d'exhortation ${ }^{33}$. Ce seront les exhortations (admonitiones) et les écrits sous la forme d'exemples (exempla) qui vont diriger notre désir (voluntas) vers la bonté, et donc vers la connaissance et l'amour de Dieu.

La rhétorique, comme la sensation, «touche légèrement » son objet; cet art touche la vérité de son objet par sa forme ; à condition que, dans l'esprit $\mathrm{du}$ vrai rhéteur, elle ne puisse pas être complètement séparée (mais radicalement distinguée) de son contenu. En citant et en réfutant le $D e$ Inventione de Cicéron (II.56.168-9), Abélard expliquera que la fin du decor, tant dans une cathédrale que dans une Écriture, n'est guère nécessaire ; elle ne fait qu'amplifier (amplificatio) ce qui est ou qui devrait être déjà là, car la vérité et la bonté ne se jouent vraiment qu'à l'intérieur, dans l'espace de l'acte et non pas de l'action externe. «C'est donc moins nos actes en euxmêmes <actions>, que l'intention avec laquelle nous les accomplissons, qu'il faut peser, si nous voulons être agréables à celui qui sonde les cœurs et les

33. P. Abélard, Commentaria in Epistolam Pauli ad Romanos, I, 1 (in : ed. E.M. Buytaert, Turnhout, Brepols, 1969. p. 8). 
reins, qui voit clair dans les ténèbres, et qui jugera les secrètes pensées des hommes, selon mon Évangile, dit saint Paul ${ }^{34}$.

La rhétorique se rapporte ainsi, de manière analogue, aux vertus dans la théorie théologique/éthique abélardienne. Le beau discours, l'apparence, l'ornementation, ou même les exempla ou exhortations, ont pour objet d'affecter, d'émouvoir, de disposer, de diriger l'homme vers le bon agir. Les vertus comme habitus, comme mises en état, sans être, en elles-mêmes, l'acte moral, le facilitent, et la rhétorique, de son côté, amplifie la vérité de son objet. Aucune des deux figures n'est suffisante pour un agir moral. Autrement dit, la rhétorique et la vertu ne se conçoivent que comme des occasions pour le bien, et non pas comme ce bien même. Mais cet état « intermédiaire » annule-t-il ces figures, l'art rhétorique et les vertus ? Certainement pas.

La rhétorique, cet outil personnel, cette persona personnifiée et impliquée dans son agir, amplifie la couche visible de la vérité. Pour reprendre une formule médiévale, la rhétorique «colore les mots ${ }^{35}$, elle peint le discours en ouvrant de nouvelles fenêtres, en offrant de nouvelles nuances pour accéder à son contenu. Autrement dit, la rhétorique diversifie les voies d'accès pour, dans la mesure du possible, atteindre la Vérité. Elle n'ajoute ni n'enlève rien à son contenu, elle n'est pas nécessaire, mais elle peut le rendre plus visible, plus évident. C'est dans cette mesure, donc, et en insistant sur la question de la persuasion, que la rhétorique est loin d'être un art méprisable. Dans son bon usage, c'est-à-dire en voulant persuader pour la compréhension de la vérité, le rhéteur rejoint le dialecticien, la persona rejoint la personne. Incapable de faire seul des miracles, l'homme doit persuader l'hérétique par le raisonnement, par la pensée - par $s a$ pensée - et sans la dialectique, fortifiée par la beauté, il est impossible de résister aux attaques des hérétiques et des infidèles et de leur fermer la bouche en leur opposant de bonnes (et belles) raisons ${ }^{36}$.

$*$

Le terme persona est divers et complexe de façon transversale. Voilà notre conclusion. Il est divers parce que la persona est différente pour le théologien, pour le grammairien et pour le rhéteur (et le comédien). Et il est complexe parce que ces différences ne sont pas complètements étrangères les unes aux autres. Ces distinctions ont précisément leur complexité, leur

34. P. Abélard, Lettre VI in V. Cousin, Lettres d'Abélard et d'Héloïse, Garnier Frères, Paris, 1875 , p. 165.

35. E. de Villena, «La Eneida de Virgilio », Revista ibérica de ciencias, politica, literatura, artes e instrucción pública, Vol. 1 (1861), p. 451.

36. Voir P. Abélard, Invective contre quelqu'un qui n'entendait rien à la dialectique, qui pourtant trouvait mauvais qu'on l'étudiât, et pensait que ses enseignements n'étaient que sophismes et tromperies, in J. Jolivet, Arts du Langage et de Théologie, p. 152. 
non-univocité, en commun: les Personnes théologiques, les personnes grammaticales, les personnes du rhéteur et du comédien ont toutes un double caractère, à savoir leur condition instrumentale (la persona est un outil) et leur trace de personnalité, de propriété. Chaque Personne théologique, chaque personne grammaticale, et chaque rhéteur (ou comédien) a quelque chose qui lui est propre : Père, Fils et Esprit Saint ; je, tu, il ; un style, une pensée sous une forme particulière.

La «personne » comme outil est «comme vide»; la personne sert à concevoir l'unité dans le multiple, elle est un agent significatif fait pour «être » l'une (des) faces du même (tant pour la Trinité que pour l'universel). Dans le cas du rhéteur, la persona est le dispositif qui nous permet, prenant une forme quelconque, d'affecter l'esprit de l'autre. Elle est «comme un maquillage », une couleur accessoire qui aide à la visibilité d'un certain contenu. Or, ni les Personnes théologiques et grammaticales, ni ce visage de l'acteur ou du rhéteur ne se trouvent complètement vidés de personnalité. Ils ont des propriétés.

Il y a quelqu'un derrière cet outil autant que devant lui. Ce nom, ce sujet et ce masque ont une particularité. On se permet d'y insister une dernière fois : toutes les personnes, dans tous les domaines, ont, à la racine de leur différence, une propriété. Elles ne peuvent pas être complètement vides, elles ne sont pas du néant; et c'est leur distinction qui leur donne, non pas une individualité, mais une personnalité. Enfin, la personne, dans sa diversité, et comme figure transversale, est fondamentale dans la pensée du dialecticien. La personne est un mot, la personne est un rôle, la personne n'existe pas, la personne est différemment, la personne a quelque chose de propre et la personne signifie. Elle affecte, elle émeut, elle engendre et elle dispose l'esprit de l'interlocuteur. Elle est l'une de voies de compréhension des plus grandes affaires de la pensée. Depuis une certaine surface - la puissance significative de cette surface -, elle nous approche de la Vérité. 